\title{
Application of Adjoint Methodology in Various Aspects of Sonic Boom Design
}

\author{
Sriram K. Rallabhandi* \\ National Institute of Aerospace, Hampton, VA 23666
}

\begin{abstract}
One of the advances in computational design has been the development of adjoint methods allowing efficient calculation of sensitivities in gradient-based shape optimization. This paper discusses two new applications of adjoint methodology that have been developed to aid in sonic boom mitigation exercises. In the first, equivalent area targets are generated using adjoint sensitivities of selected boom metrics. These targets may then be used to drive the vehicle shape during optimization. The second application is the computation of adjoint sensitivities of boom metrics on the ground with respect to parameters such as flight conditions, propagation sampling rate, and selected inputs to the propagation algorithms. These sensitivities enable the designer to make more informed selections of flight conditions at which the chosen cost functionals are less sensitive.
\end{abstract}

\section{Nomenclature}

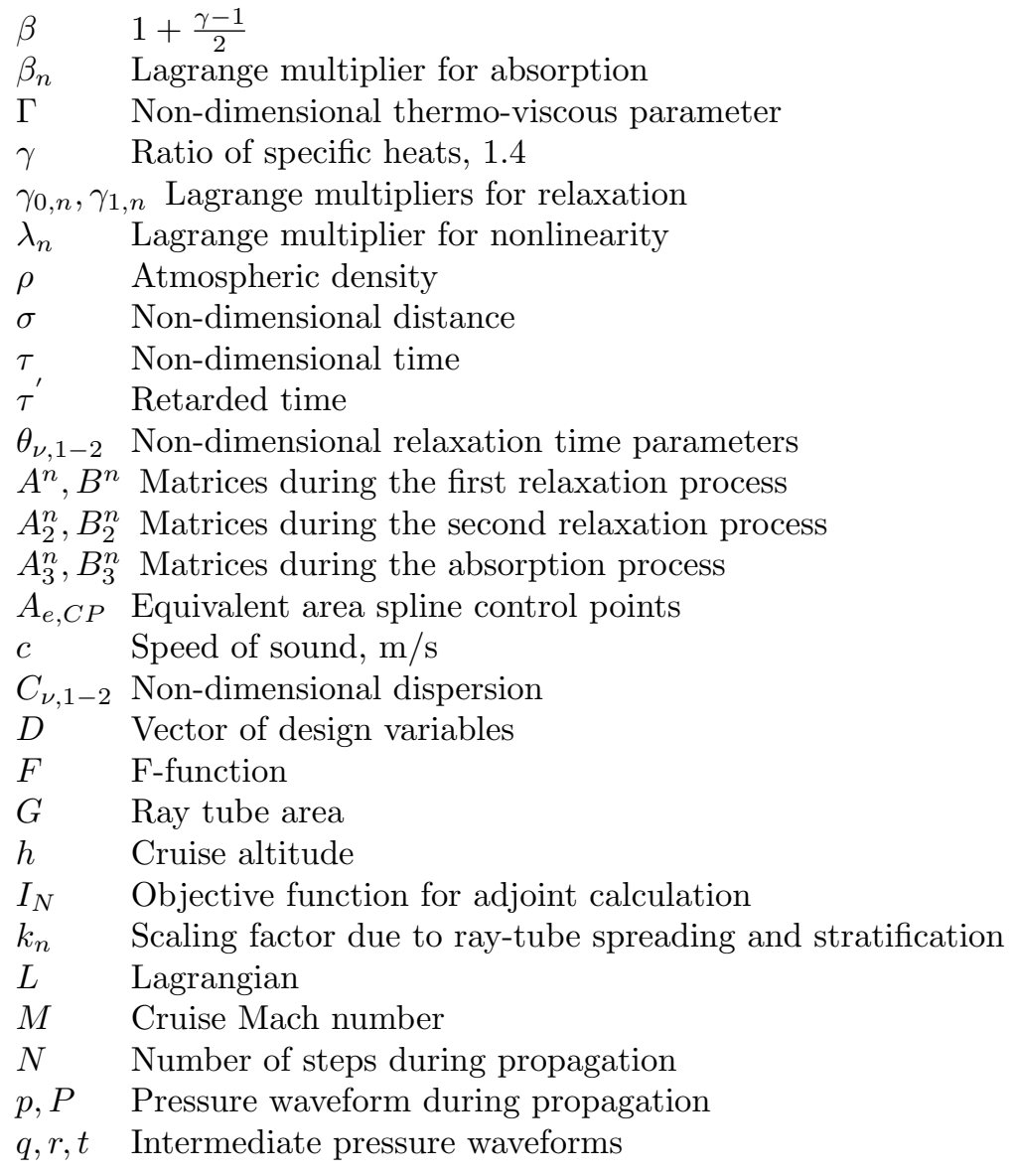

${ }^{*}$ Senior Research Engineer, National Institute of Aerospace, Associate Fellow AIAA 
$S \quad$ Number of points used in propagation

$X_{C P} \quad$ Axial spline control points

Subscripts

$0 \quad$ Starting conditions

$n \quad$ Propagation iteration counter

\section{Introduction and Motivation}

There has been a lot of interest for a viable commercial supersonic aircraft after the successful demonstration of the DARPA Shaped Sonic Boom Demonstrator (SSBD) ${ }^{1}$ program. To mitigate the adverse effects of sonic boom, several efforts ${ }^{2,3,4,5,6,7}$ have been carried out to design aircraft that overcome stringent acceptability constraints that currently forbid overland supersonic flight. Significant advances in computational design in terms of tools and methodologies have been developed that complement the existing body of knowledge from the past several decades. One of the first steps in this design process is developing a framework that allows designers to explore the design space efficiently and develop concepts using appropriate analysis fidelity. In addition to non-gradient optimization methods, computational approaches based on sensitivities offer an elegant way to reduce sonic boom levels. Adjoint-based methods in particular provide an efficient way to compute sensitivities of chosen metrics to a large number of shape design parameters. Previous studies have demonstrated the capability of adjoint-based methods to optimize near-field pressure waveforms,${ }^{8,2,9}$ ground-based boom metrics, ${ }^{10}$ as well as equivalent area metrics. ${ }^{5,11}$

The methodology described in this study builds upon the adjoint methodology from previous papers ${ }^{10,11}$ and extends the adjoint formulation in $\mathrm{sBOOM}^{12}$ it in two ways. The first extension allows generation of equivalent area targets from sensitivity information which enables designers to quickly develop smooth targets for under-track and off-track azimuths in the neighborhood of the baseline design. The second extension calculates adjoint sensitivities of boom acceptability metrics to flight conditions (e.g., Mach, cruise altitude), propagation parameters (absorption and relaxation constants, step-size), and sampling rate used during propagation. This work facilitates uncertainty analysis and robust design of low-boom aircraft.

\section{Extension to Existing Theory}

The underlying mathematics behind boom adjoints is similar to that presented in Refs. 10 and 11. In this paper, we focus only on specific details that pertain to target equivalent area generation and sensitivities with respect to atmospheric and propagation parameters.

\section{II.A. Target equivalent area generation}

The theory behind computation of sensitivities needed in this case is a simple extension of the theory presented in Ref. 10. The primary difference is that the independent design variable vector is the input equivalent area instead of the off-body pressure waveform. To extend the sensitivities to equivalent area, we employ the chain rule of differentiation as shown in Eq. 1, where the relation $d p / p=\frac{\gamma M^{2}}{\sqrt{2 \beta R}} F$ is used. The first term on the right hand side is calculated from the previous formulation. The F-function $(F)^{13}$ is calculated from equivalent area $\left(A_{e}\right)$ as given in Eq. 2. Numerically differentiating this relation yields $\frac{d F}{d A e}$, which can be used in Eq. 1 to obtain the relevant sensitivity of the cost functional with respect to the equivalent areas.

$$
\begin{gathered}
\frac{d L}{d A e}=\frac{d L}{d(d p p)} \frac{d(d p p)}{d A e}=\frac{d L}{d(d p p)} \frac{\gamma M^{2}}{\sqrt{2 \beta R}} \frac{d F}{d A e} \\
F(y)=\frac{1}{2 \pi} \int_{0}^{y} \frac{A e^{\prime \prime}}{(y-x)^{1 / 2}} d x
\end{gathered}
$$

The objective chosen to minimize is the A-weighted loudness of the ground signature. While it is desirable to use the perceived loudness level as the objective during optimization, there was no easy way to compute the sensitivity of this metric with respect to the ground signature that is needed in adjoint-based design optimization. A-weighted loudness computation, on the other hand, offers an analytical calculation procedure 
that can be differentiated for generating required sensitivities and therefore amenable to adjoint-based design. In addition, the A-weighted loudness is well correlated ${ }^{14}$ with the perceived loudness values. If a technique were to become available that would allow computation of perceived loudness level sensitivities, then the current methodology could be extended to minimize a cost functional that is a function of the perceived loudness of the sonic boom ground signature.

At this point, a gradient-based optimizer can be used to change the baseline equivalent area values at different longitudinal sections along the aircraft to minimize the A-weighted loudness. However, this optimization can lead to equivalent area shapes that are not smooth. This is because the optimizer exploits shock cancellation in a favorable way due to the nature of the propagation algorithm. This is demonstrated in Figures 1, 2 and 3. Figure 1 depicts an optimized equivalent area that is visually smooth, but has ripples throughout when closely inspected. Looking at the equivalent area in F-function space reveals that the equivalent area ripples manifest as large oscillations around a smooth profile. During propagation, these oscillations cancel one another and the resulting ground signature improves relative to the baseline (See Fig. 3) as predicted by the optimizer.

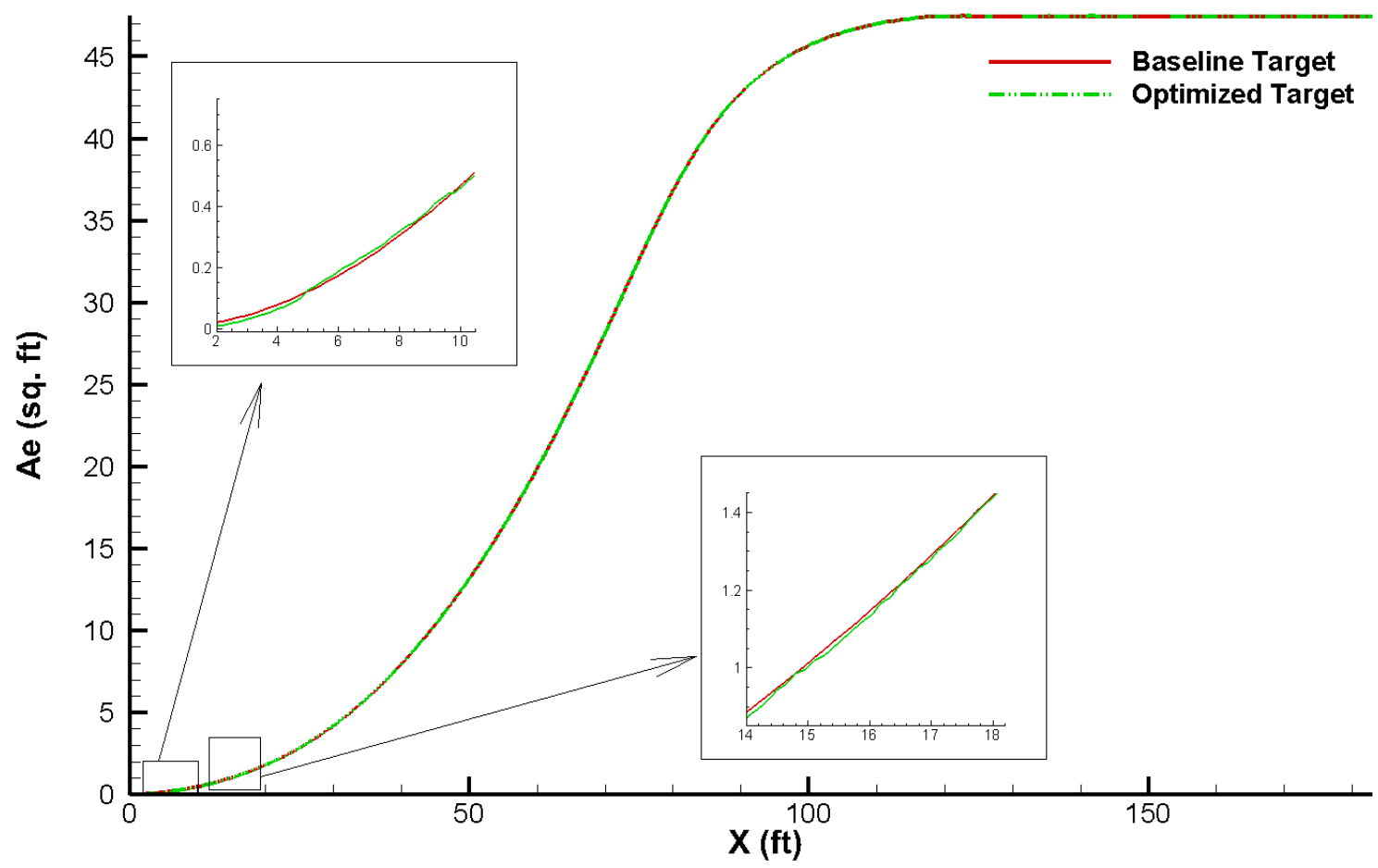

Figure 1: An example of non-smooth target generated without spline smoothing.

While non-smooth targets are numerically better, designers seek smooth targets not only for better performance but also for improved robustness in the final designs. To overcome non-smoothness in the target equivalent areas, an additional step is needed wherein smooth equivalent area shapes are generated by manupulating the control points of a spline. A similar approach using Bezier curves defined by arbitrary number of control points to represent the target equivalent area distributions is employed in Ordaz et al. ${ }^{15}$ One difference here is that the spline-based algorithm not only returns the spline interpolation but also produces the Jacobian matrices $\frac{\partial A_{e}}{\partial X_{C P}}$ and $\frac{\partial A_{e}}{\partial A_{e}, C P}$. These matrices are then used in Eq. 3 to produce the sensitivity of the objective functions to the spline control points. In this paper, we generate a cubic spline and the corresponding Jacobian matrices. However, the formulation can be extended to other curve fitting approaches.

$$
\frac{d L}{d D}= \begin{cases}\frac{d L}{d A_{e}} \frac{d A_{e}}{d X_{C P}} & \text { if } D=X_{C P} \\ \frac{d L}{d A_{e}} \frac{d A_{e}}{d A_{e}, C P} & \text { if } D=A_{e, C P}\end{cases}
$$




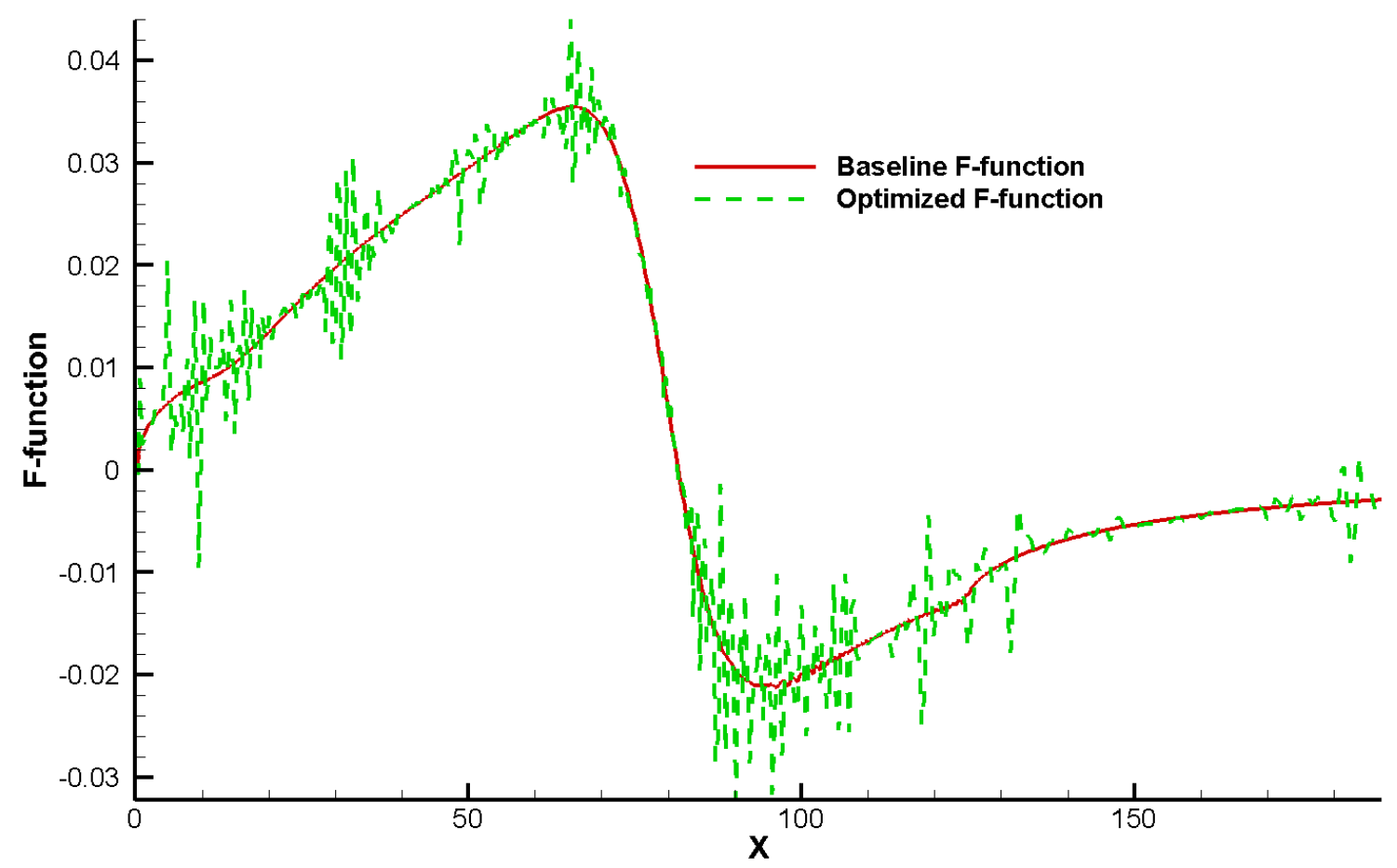

Figure 2: An example of non-smooth F-function generated without spline smoothing.

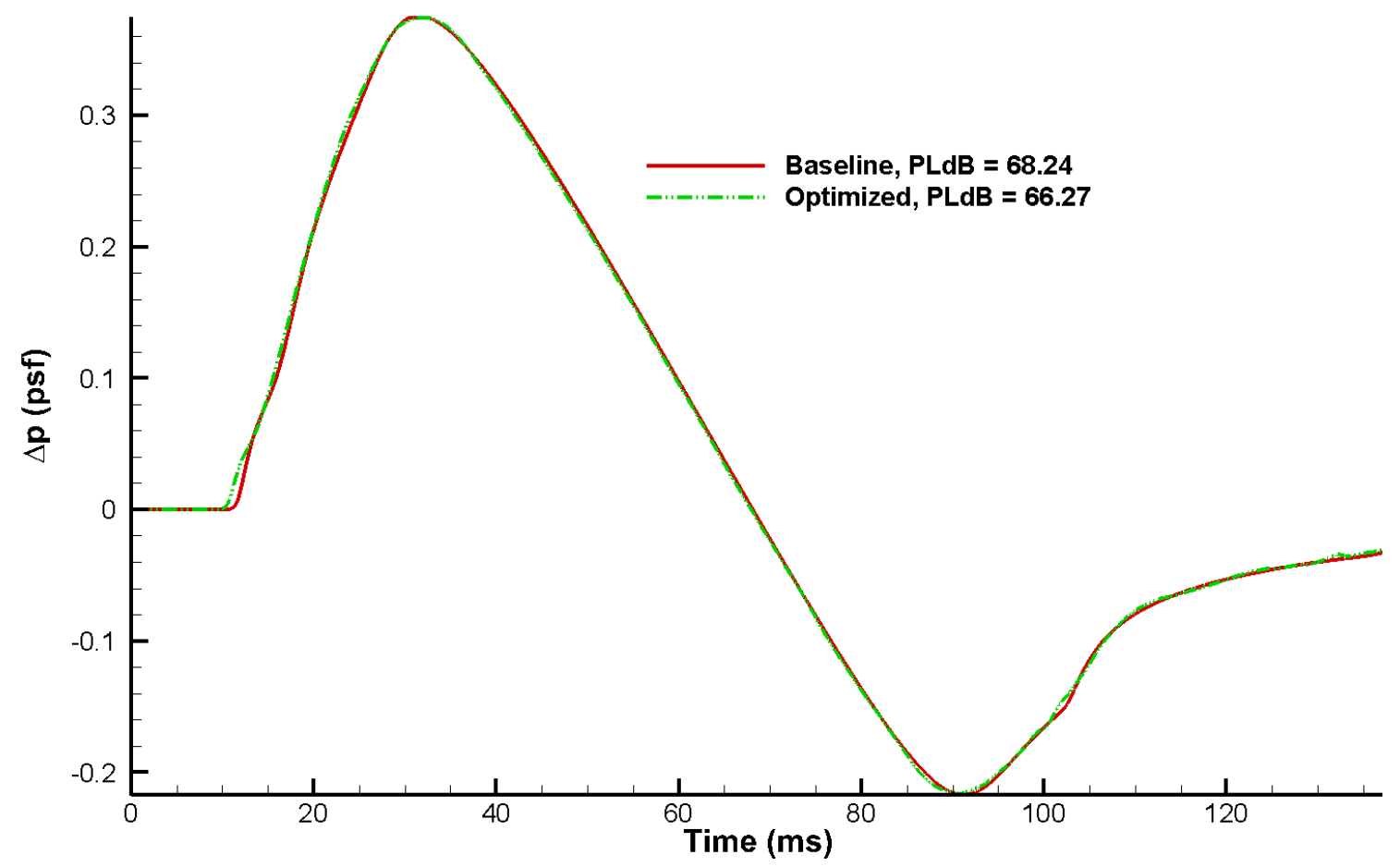

Figure 3: An example demonstrating numerically better ground signature without spline smoothing. 


\section{II.B. Sensitivities with respect to flight conditions and propagation parameters}

The discrete adjoint equations derived in this section are based on a similar implementations developed in Ref. 10. The primary difference is the independent variable vector $\mathrm{D}$, which has been modified to represent the 9-tuple vector $\left[\Delta \sigma, S, \Gamma, \theta_{\nu, 1}, C_{\nu, 1}, \theta_{\nu, 2}, C_{\nu, 2}, h, M\right]$. The formulation is not limited to this vector; other variables such as temperature and wind profiles may also be included. However, in this study we are limiting ourselves to the nine variables listed.

The Lagrangian corresponding to a chosen objective may be written as in Eq. 4, where $k_{n}$ is the scaling factor given by $\sqrt{\frac{\rho_{0} c_{0} G}{\rho c G_{0}}}$. The details of the matrix terms are provided in the Appendix. Taking the derivative of the Lagrangian with respect to $D$ results in Eq. 5. Contrary to the previous formulations, the relevant matrices during propagation now depend on the design variable vector $D$ and hence the matrix derivative terms now appear in the derivative of theLagrangian.

$$
\begin{gathered}
L(p, q, r, t, D)=I_{N}\left(p_{N}, D\right)+\sum_{n=1}^{N} \gamma_{0, n}^{T}\left[A^{n} q_{n}-k_{n} B^{n} p_{n-1}\right]+\sum_{n=1}^{N} \gamma_{1, n}^{T}\left[A_{2}^{n} r_{n}-B^{n} q_{n}\right] \\
+\sum_{n=1}^{N} \beta_{n}^{T}\left[A_{3}^{n} t_{n}-B_{3}^{n} r_{n}\right]+\sum_{n=1}^{N} \lambda_{n}^{T}\left[p_{n}-f^{n}\left(t_{n}, D\right)\right] \\
\frac{d L}{d D}=\left[\frac{\partial I_{N}}{\partial D}+\frac{\partial I_{N}}{\partial p_{N}} \frac{\partial p_{N}}{\partial D}\right]+\sum_{n=1}^{N} \gamma_{0, n}^{T}\left[A^{n} \frac{\partial q_{n}}{\partial D}+\frac{\partial A^{n}}{\partial D} q_{n}-k_{n} B^{n} \frac{\partial p_{n-1}}{\partial D}-\frac{\partial k_{n}}{\partial D} B^{n} p_{n-1}-k_{n} \frac{\partial B^{n}}{\partial D} p_{n-1}\right] \\
+\sum_{n=1}^{N} \gamma_{1, n}^{T}\left[A_{2}^{n} \frac{\partial r_{n}}{\partial D}+\frac{\partial A_{2}^{n}}{\partial D} r_{n}-B_{2}^{n} \frac{\partial q_{n}}{\partial D}-\frac{\partial B_{2}^{n}}{\partial D} q_{n}\right]+\sum_{n=1}^{N} \beta_{n}^{T}\left[A_{3}^{n} \frac{\partial t_{n}}{\partial D}+\frac{\partial A_{3}^{n}}{\partial D} t_{n}-B_{3}^{n} \frac{\partial r_{n}}{\partial D}-\frac{\partial B_{3}^{n}}{\partial D} r_{n}\right] \\
+\sum_{n=1}^{N} \lambda_{n}^{T}\left[\frac{\partial p_{n}}{\partial D}-\frac{\partial f_{j}^{n}}{\partial t_{n}} \frac{\partial t_{n}}{\partial D}\right]
\end{gathered}
$$

The adjoint equations and their solution procedure are identical to previous formulations and are not repeated here. Once the adjoint terms are removed, the adjoint gradient can be calculated using the expression in Eq. 6. It should be noted that the matrix Jacobian terms are three-dimensional matrices. It is clear by looking at this equation that the memory requirement increases significantly from the previous formulation. This is due to multiple matrix Jacobians and intermediate pressure waveforms that need to be stored at each step during the propagation process in addition to the Lagrange multipliers. Sensitivity of $k_{n}$ and consequently the sensitivity of the Blokhintzev invariant (See Eq. 7, in the absence of winds) ${ }^{16,17}$ also needs to computed and stored.

$$
\begin{gathered}
\frac{d L}{d D}=\sum_{n=1}^{N} \gamma_{0, n}^{T}\left[\frac{\partial A^{n}}{\partial D} q_{n}-\frac{\partial k_{n}}{\partial D} B^{n} p_{n-1}-k_{n} \frac{\partial B^{n}}{\partial D} p_{n-1}\right] \\
+\sum_{n=1}^{N} \gamma_{1, n}^{T}\left[\frac{\partial A_{2}^{n}}{\partial D} r_{n}-\frac{\partial B_{2}^{n}}{\partial D} q_{n}\right]+\sum_{n=1}^{N} \beta_{n}^{T}\left[\frac{\partial A_{3}^{n}}{\partial D} t_{n}-\frac{\partial B_{3}^{n}}{\partial D} r_{n}\right] \\
p \sqrt{\frac{G}{\rho c}}=\text { constant }
\end{gathered}
$$

The matrix Jacobians can be obtained by differentiating the individual terms of the matrices in the Appendix. For sake of brevity, only a few of these are shown in the Appendix. 


\section{Verification of Adjoint Sensitivities}

To verify that the sensitivity values obtained from adjoint calculations are correct, a complex variable version of the process is developed. The use of complex step approach ${ }^{18,19}$ is standard practice ${ }^{20}$ for gradient verification. The relevant algorithms are modified to work with complex variables and the derivatives of appropriate cost functionals with respect to the chosen design variables are calculated using an imaginary step size of $10^{-50}$.

\section{III.A. Target equivalent area generation}

Table 1 shows a comparison of the A-weighted loudness sensitivity to equivalent area values at selected indices, computed using the adjoint and complex versions of the code. It can be seen that the values agree to 9 decimal places, verifying that the sensitivity values are accurately computed.

III.B. Sensitivities with respect to flight conditions and propagation parameters

Table 2 compares the adjoint and complex variable gradients of ground loudness with respect to each of the flight condition and propagation parameters. The results obtained using the adjoint implementation exhibit good agreement with the complex-variable approach. Contrary to previous formulations, this sensitivity calculation involves summation over the entire propagation distance, causing some precision loss in the sensitivity comparisons. Nevertheless, the adjoint sensitivities are reliable for any subsequent analysis.
Table 1: Comparison of the adjoint and complex variable gradients for $A_{e}$ sensitivity.

\begin{tabular}{ccc}
\hline \hline Grid Point & Adjoint Gradient & Complex Gradient \\
\hline 0 & 4.99555122854739 & $4.99555122 \underline{485994}$ \\
200 & 0.22333517023795 & $0.223335170 \underline{026696}$ \\
500 & -1.83336776546880 & $-1.83336776 \underline{671720}$ \\
\hline
\end{tabular}

Table 2: Comparison of the adjoint and complex variable gradients for flight and propagation parameters.

\begin{tabular}{ccc}
\hline \hline Variable & Adjoint Gradient & Complex Gradient \\
\hline$S$ & $-0.121609572658 \mathrm{E}-002$ & $-0.121609 \underline{679831 \mathrm{E}-002}$ \\
$\Delta \sigma$ & $-0.101433622238 \mathrm{E}+004$ & $-0.10143 \underline{4351727} \mathrm{E}+004$ \\
$\Gamma$ & $-0.242603369041 \mathrm{E}+005$ & $-0.24260 \underline{7576473} \mathrm{E}+005$ \\
$C_{\nu_{1}}$ & $0.850297505015 \mathrm{E}+002$ & $0.85029 \underline{3279821} \mathrm{E}+002$ \\
$C_{\nu_{2}}$ & $0.114275582996 \mathrm{E}+003$ & $0.11427 \underline{9749352} \mathrm{E}+003$ \\
$\theta_{\nu_{1}}$ & $-0.270434382922 \mathrm{E}+004$ & $-0.27043 \underline{8763849} \mathrm{E}+004$ \\
$\theta_{\nu_{2}}$ & $-0.226512310051 \mathrm{E}+005$ & $-0.2265 \underline{\underline{34988755} \mathrm{E}+005}$ \\
$M$ & $0.103286198407 \mathrm{E}+002$ & $0.1032 \underline{1674303} \mathrm{E}+002$ \\
$h$ & $0.373155218390 \mathrm{E}-004$ & $0.3731552 \underline{85384} \mathrm{E}-004$ \\
\hline \hline
\end{tabular}

\section{Results}

\section{IV.A. Generation of target equivalent area}

In this section, a gradient-based optimization process of generating smooth equivalent area targets is demonstrated. A baseline concept is evaluated at multiple azimuthal angles and its off-body pressure waveform and the corresponding area distributions obtained. Using the end areas, a cubic spline is fit to the equivalent areas at each of the azimuthal angles. In this demonstration, 15 control points are used for each spline fit. An SQP algorithm ${ }^{21}$ was used as the gradient-based optimizer. Figure 4 depicts the design and target equivalent areas for each of the azimuthal angles. Smooth targets are generated for each azimuthal angle in the neighborhood of the underlying design area.

A typical convergence history of the optimizer is shown in Fig. 5. Convergence is achieved in 50-60 function evaluations, taking roughly 30 minutes of wall-clock time on a 64-bit Intel Xeon processor with 16GB of memory. Targets at different azimuthal angles can be obtained simultaneously allowing designers 




Figure 4: Generation of targets via gradient-based optimization using adjoint sensitivities.

to quickly generate equivalent area targets. In addition, because the targets are in the neighborhood of the underlying areas at each of the azimuthal angles, there is a high degree of correlation between the targets. This can be helpful during shape optimization to make the problem better posed for obtaining an outer mold line that can match the under- and off-track targets simultaneously. Because of the fast turn around time, targets can be updated as the design evolves. The ground signatures corresponding to the generated targets are shown in Fig. 6.

\section{IV.B. Sensitivity to flight conditions and propagation parameters}

Figure 7 shows the sensitivity of the A-weighted loudness to varying flight conditions and some propagation parameters. It is to be noted that these plots are dependent on the underlying off-body pressure waveform, and hence dependent on the concept under investigation. Some of the trends may be different for other near-field waveforms. Several observations may be made from these plots.

1. The sensitivities with respect to Mach number and step size $(\Delta \sigma)$ are much larger than for cruise altitude and number of points used in propagation. Sensitivity with respect to Mach number decreases as Mach number increases. This is perhaps because as the Mach number increases, the ground signature tends to an N-wave. Put another way, the sensitivity is greater when the ground signature is shaped and decreases as the signature tends towards $\mathrm{N}$-waves.

2. According to the US standard atmosphere, ${ }^{22}$ a constant lapse rate exists below 36,089 feet, which causes the Blokhintzev invariant to vary as the cruise altitude changes. Above this altitude, in the tropopause, cruise altitude variations do not change the ambient density or the Blokhintzev invariant. Thus, above 36,089 feet, the sensitivity becomes essentially zero.

3. As the number of points used in propagation increases, the loudness sensitivity asymptotically goes to zero, the loudness values converge while the computational time increases. This is a parameter where the sensitivity and uncertainty go down simultaneously.

4. For small step sizes, sensitivity levels are high. As the step size increases, a point is reached where the propagation algorithm determines that the user provided step size is too large to avoid multi-valued pressure profiles, and hence picks the largest step size that allows the propagation to run. At this step size, the sensitivity value goes to zero. However, the error in loudness might be large because as the step size increases, the error induced also increases at each step and the cumulative effect during propagation to the ground can be significant. 




Figure 5: Typical convergence history of the gradient optimizer.

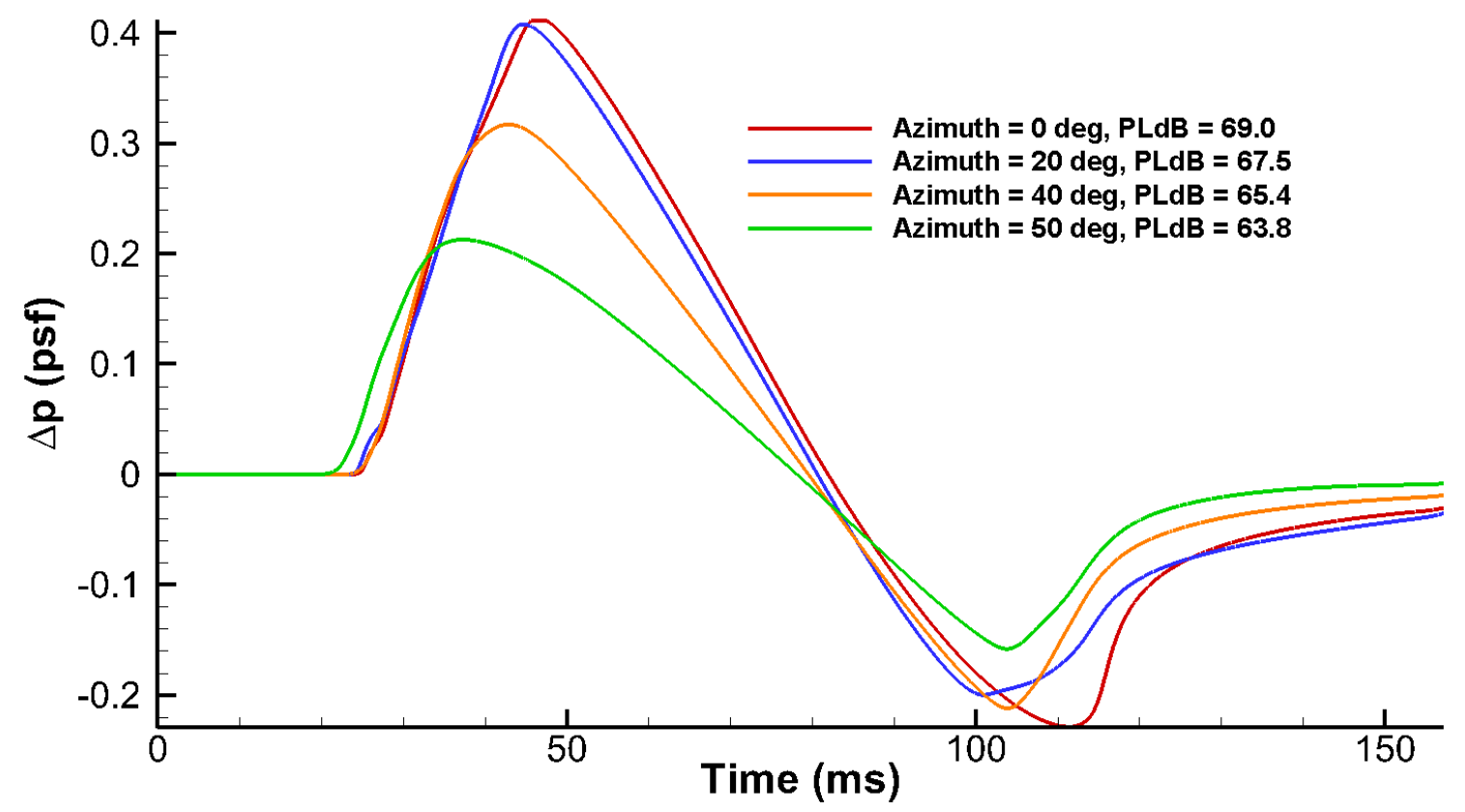

Figure 6: Ground signatures corresponding to targets.

Figure 8 shows the sensitivity of the scaling factor $k_{n}$ to Mach number at different conditions. The primary take-away from this plot is that the scaling factor sensitivity, and consequently the ray tube area sensitivity is small at smaller step sizes compared to larger step sizes.

This paper considered only some sources of epistemic uncertainty. One possible extension of this work is to include sources of aleatory uncertainty such as wind, temperature and relative humidity profiles during atmospheric propagation. The foregoing analysis and its associated sensitivities may be used to quantify uncertainty during the propagation process. Uncertainty analysis could either be sampling-based, such as Monte-Carlo analysis, or non-sampling-based, such as polynomial chaos. Whatever method is chosen, the design intent is to reach a robust point where the error as well as sensitivity are simultaneously minimized. Even if such a point cannot be reached, the information obtained could be valuable in the understanding and exploration of the design space. 


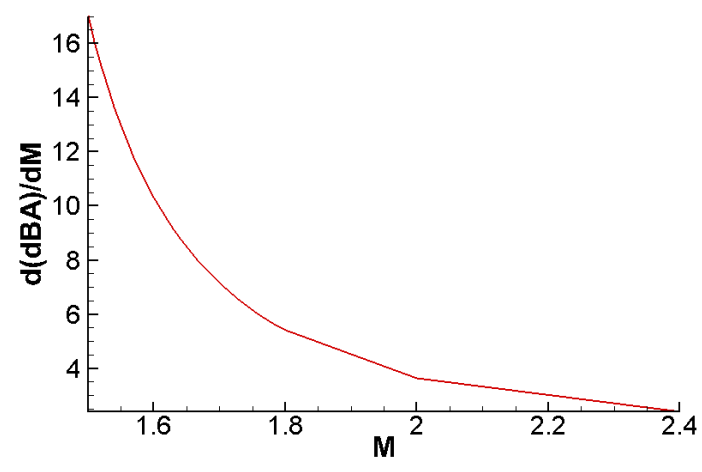

(a) Loudness sensitivity wrt $M$.



(c) Loudness sensitivity wrt $S$.

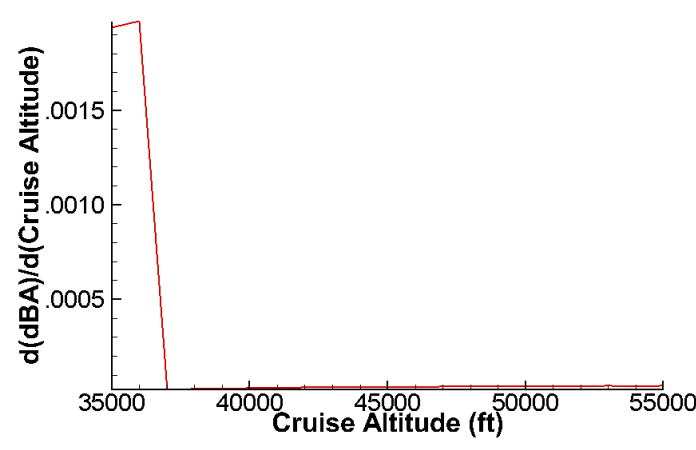

(b) Loudness sensitivity wrt $h$.



(d) Loudness sensitivity wrt $\Delta \sigma$.

Figure 7: Sensitivities of flight conditions and selected propagation parameters.

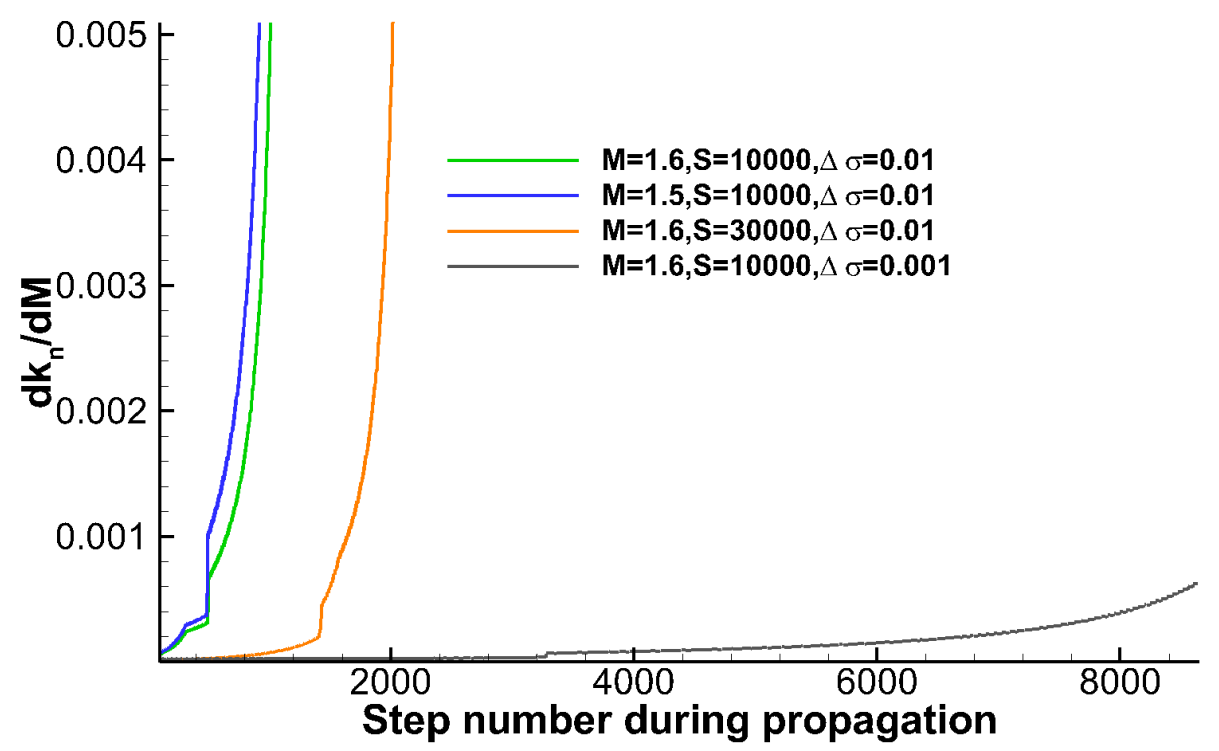

Figure 8: Sensitivities of scaling factor to Mach number. 


\section{Conclusions}

The adjoint formulation within the sBOOM propagation has been extended to generate sensitivities with respect to equivalent areas, flight conditions and selected propagation parameters. Equivalent area targets were generated using a gradient-based optimizer at multiple azimuthal angles that minimize ground loudness at each off-track location. These may be used during shape optimization exercises for matching at under- and off-track locations. The sensitivity of the loudness to flight conditions and propagation parameters has been plotted and observations have been made. As the design evolves into the preliminary design stage, analysis using sensitivities with respect to flight conditions, propagation parameters and atmospheric properties may be used to understand the uncertainty of the underlying propagation analysis as well as provide feedback to steer towards a robust design.

\section{Appendix}

The tridiagonal matrices for the relaxation processes are:

$$
\begin{aligned}
& A^{n}, A_{2}^{n}=\left(\begin{array}{cccccc}
1 & 0 & \cdots & & \\
0 & 1 & 0 & \ldots & & \\
0 & -\alpha \kappa_{1}-\kappa_{2} & \left(1+2 \alpha \kappa_{1}\right) & \kappa_{2}-\alpha \kappa_{1} & \ldots & \\
& \ddots & \ddots & \ddots & & \\
& & \cdots & 0 & 1 & 0 \\
& & \ldots & & 0 & 1
\end{array}\right) \\
& B^{n}, B_{2}^{n}=\left(\begin{array}{cccccc}
1 & 0 & \cdots & & \\
0 & 1 & 0 & \cdots & \\
\alpha^{\prime} \kappa_{1}-\kappa_{2} & \left(1-2 \alpha^{\prime} \kappa_{1}\right) & \kappa_{2}+\alpha^{\prime} \kappa_{1} & \cdots & \\
& \ddots & \ddots & \ddots & & \\
& & \cdots & 0 & 1 & 0 \\
& & \cdots & & 0 & 1
\end{array}\right)
\end{aligned}
$$

In the above matrices, $\kappa_{1}=\frac{C_{\nu} \Delta \sigma_{n}}{\Delta \tau^{2}}, \kappa_{2}=\frac{\theta_{\nu}}{2 \Delta \tau}$, and $\alpha^{\prime}=1-\alpha$. If using the Crank-Nicholson scheme, $\alpha=0.5$. For thermo-viscous absorption, the matrices are given below with $\lambda=\frac{\Delta \sigma_{n}}{2 \Gamma(\Delta \tau)^{2}}$

$$
\begin{gathered}
A_{3}^{n}=\left(\begin{array}{ccccc}
1 & 0 & \cdots & \\
-\lambda & (1+2 \lambda) & -\lambda & \cdots & \\
& \ddots & \ddots & \ddots & \\
& & \cdots & 0 & 1
\end{array}\right) \\
B_{3}^{n}=\left(\begin{array}{ccccc}
1 & 0 & \cdots & \\
\lambda & (1-2 \lambda) & \lambda & \cdots & \\
& \ddots & \ddots & \ddots & \\
\frac{\partial \kappa_{1}}{\partial \Delta \tau}=-\frac{2 C_{\nu} \Delta \sigma_{n}}{\Delta \tau^{3}} ; \quad & \cdots & 0 & 1
\end{array}\right) \\
\frac{\partial \kappa_{1}}{\partial \Delta \sigma}=\frac{C_{\nu}}{\Delta \tau^{2}} ; \quad \frac{\partial \kappa_{2}}{\partial \Delta \tau}=-\frac{\theta_{\nu}}{2 \Delta \tau^{2}} \\
\frac{\partial \kappa_{1}}{\partial C_{\nu}}=-\frac{\Delta \sigma_{n}}{\Delta \tau^{2}} \\
\frac{\partial \lambda}{\partial \Delta \tau}=-\frac{\Delta \sigma_{n}}{\Gamma \Delta \tau^{3}}
\end{gathered} \quad \quad \frac{\partial \kappa_{2}}{\partial \theta_{\nu}}=\frac{1}{2 \Delta \tau}
$$




\section{Acknowledgments}

This work was supported by the NASA Project entitled "Sonic Boom Propagation Tools and Methods for Low Sonic Boom Design," under NASA contract number NNL13AA08B, task number NNL13AC78T through the NASA Fundamental Aeronautics High Speed Program. The author wishes to thank Jim Fenbert for setting up a working ModelCenter model running a gradient-based optimizer for target equivalent area generation. Help in several forms from Karl Geiselhart (ModelCenter), Irian Ordaz (off-track analysis), Mathias Wintzer, Lori Ozoroski and Wu Li is gratefully acknowledged.

\section{References}

${ }^{1}$ Pawlowski, J. W., Graham, D. H., et al., "Origins and Overview of the Shaped Sonic Boom Demonstration Program," AIAA Paper 2005-5, Jan. 2005.

${ }^{2}$ Aftosmis, M., Nemec, M., and Cliff, S., "Adjoint-based Low-Boom Design with Cart3D (Invited)," AIAA Paper No. 2011-3500, 2011.

${ }^{3}$ Howe, D., Simmons, F., and Freund, D., "Development of the Gulfstream Quiet Spike TM for Sonic Boom Minimization," AIAA Paper 2008-0124, 2008.

${ }^{4}$ Morgenstern, J., Norstrud, N., Stelmack, M., and Skoch, C., "Final Report for the Advanced Concept Studies for Supersonic Commercial Transports Entering Service in the 2030 to 2035 Period, N+3 Supersonic Program,", Tech. Rep. CR-2010216796, PMF-01623, E-17427,Document ID 20100036507, National Aeronautics and Space Administration, Langley, Virginia, Oct. 2010.

${ }^{5}$ Francisco Palacios, Juan J. Alonso, M. C. J. H. and Lukaczyk, T., "Adjoint-Based method for supersonic aircraft design using equivalent area distribution," AIAA Paper No. 2012-0269, 2012.

${ }^{6}$ Magee, T. E., Shaw, S. G., and Fugal, S. R., "Experimental Validations of a Low-Boom Aircraft Design," AIAA Paper No. 2013-0646, 2013.

${ }^{7}$ Morgenstern, J. M., "Tail-braced wing aircraft and configurations for achieving long supersonic range and low sonic boom," US Patent No. 6729577, Lockheed Martin Corporation, May 2004.

${ }^{8}$ Nadarajah, S., Jameson, A., and Alonso, J., "Sonic Boom Reduction using an Adjoint Method for Wing-Body Configurations in Supersonic Flow," AIAA Paper No. 2002-5547, 2002.

${ }^{9}$ Wintzer, M. and Kroo, I., "Optimization and Adjoint-Based CFD for the Conceptual Design of Low Sonic Boom Aircraft," AIAA Paper No. 2012-0963, 2012.

${ }^{10}$ Rallabhandi, S. K., Nielsen, E. J., and Diskin, B., "Sonic-Boom Mitigation Through Aircraft Design and Adjoint Methodology," Journal of Aircraft, Vol. 51, No. 2, 2014, pp. 502-510.

${ }^{11}$ Rallabhandi, S. K., "Application of Adjoint Methodology to Supersonic Aircraft Design Using Reversed Equivalent Areas," AIAA Paper No. 2013-2663, 2013.

${ }^{12}$ Rallabhandi, S. K., "Advanced Sonic Boom Prediction Using Augmented Burger's Equation," Journal of Aircraft, Vol. 48, No. 4, 2011, pp. 1245-1253.

${ }^{13}$ Whitham, G., "The Flow Pattern of a Supersonic Projectile," Communications on Pure and Applied Mathematics, Vol. V, 1952, pp. 301-347.

${ }^{14}$ Sullivan, B. M., Klos, J., Buehrle, R. D., McCurdy, D. A., and Edward A. Haering, J., "Human Response to Low-Intensity Sonic Booms Heard Indoors and Outdoors," NASA TM 2010-216685, 2010.

${ }^{15}$ Ordaz, I., Geiselhart, K. A., and Fenbert, J. W., "Conceptual Design of a Low-Boom Demonstrator with Flight Trim Requirement," AIAA Paper No. 2014-xxxx, 2014.

${ }^{16}$ Thomas, C., "Extrapolation of sonic boom pressure signatures by the waveform parameter method," Tech. Rep. NASA TN D-6832, NASA, June 1972.

${ }^{17}$ Plotkin, K. J., "Review of Sonic Boom Theory," AIAA Paper No. 89-1105, 1989.

${ }^{18}$ Lyness, J. N., "Numerical Algorithms Based on the Theory of Complex Variables," Proceedings of the ACM 22nd National Conference, 1967, pp. 124-134.

${ }^{19}$ Lyness, J. N. and Moler, C. B., "Numerical Differentiation of Analytic Functions," SIAM Journal on Numerical Analysis, Vol. 4, 1967, pp. 202-210.

${ }^{20}$ Nielsen, E. and Diskin, B., "Discrete Adjoint-Based Design for Unsteady Turbulent Flows on Dynamic Overset Unstructured Grids," AIAA Paper No. 2012-0554, 2012.

${ }^{21}$ Lawrence, C. T., Zhou, J. L., and Tits, A. L., "User's Guide for CFSQP Version 2.4: A C Code for Solving (Large Scale) Constrained Nonlinear (Minimax) Optimization Problems, Generating Iterates Satisfying All Inequality Constraints," Tech. Rep. Technical Report TR-94-16r1, Institute for Systems Research, University of Maryland, College Park, MD, 1996.

22 "U.S. Standard Atmosphere," U.S. Government Printing Office, Washington, D.C., 1976. 